\title{
Improvement of an Algorithm for Estimation of Reaction Torque and Compensation for Elongation of Wire for Wire-Actuated Robotic Forceps
}

\author{
Chiharu Ishii and Gakuto Komada
}

\begin{abstract}
When a load is added to grasping jaws of a wire-actuated robotic forceps, tracking accuracy of the position of the grasping jaws is degraded due to the elastic elongation of the wire. In the previous work, we have proposed an algorithm that estimates a reaction torque loading the grasping jaws of wire-actuated robotic forceps and compensates for subsequent wire elongation. However, our previous algorithm required an assumption that the motor torque and reaction torque are regarded as constant, although they fluctuate in practice. This paper proposes a new algorithm that eliminates this assumption, resulting much improved accuracy in estimating the reaction torque. The new algorithm is constructed by the combination of the reaction force observer (RFOB) and an optimization problem based on a gradient descent method. The improvement was verified through simulation.
\end{abstract}

Index Terms-Reaction force observer (RFOB), wire-actuated robotic forceps, estimation of reaction torque, compensation for wire elongation.

\section{INTRODUCTION}

Recently, minimally invasive surgery has contributed to dramatic progress in medical treatment. In addition, robotic surgical support systems, such as da Vinci [1] produced by Intuitive Surgical Inc., have increasingly been applied in clinical practice worldwide. Similar to da Vinci, most multi-DOF robotic forceps have a wire-actuated mechanism; e.g., [2], [3]. However, for the wire-actuated robotic forceps, a decrease in tracking accuracy owing to the elastic deformation of the wire is an inevitable [4]. Even for skilled surgeons, tracking accuracy of grasping jaws of the forceps is important, since delicate operation is required for a fingertip work. Therefore, many studies have been tried to enhance the tracking accuracy of grasping jaws of the forceps; e.g., [5], [6].

On the other hand, force feedback technology for the robotic forceps has been studied for many years, since the lack of tactile perception may cause serious injury. The most straightforward way to achieve force feedback is to install sensors on the robotic forceps. Haptic forceps manipulator for minimally invasive surgery has been proposed in [7]-[9], in which external force is measured by sensor and force feedback is provided.

Manuscript received December 1, 2014; revised January 15, 2015. This study was partially supported by Grants-In-Aid for Scientific Research (C) 25330312 .

Chiharu Ishii is with the Department of Mechanical Engineering, Hosei University, Tokyo 184-8584, Japan (e-mail: c-ishii@hosei.ac.jp).

Gakuto Komada is with the Graduate School of Engineering, Hosei University, Tokyo 184-8584, Japan (e-mail: gakuto.komada.3v@stu.hosei.ac.jp).
However, it is not preferable to install sensors on a part that enters the patient's body from hygienic and safety points of view. Thus, it is believed that a study on the estimation of the reaction torque loading the grasping jaws of the forceps without using force/torque sensors and compensating for the amount of wire elongation will contribute to the further development of a robotic surgical system.

To the best of our knowledge, estimation of the reaction torque for wire-actuated robotic forceps with elongation of the wire has hardly been studied. In our previous study [10], we proposed an algorithm that estimates a reaction torque and elongation of the wire. However, this algorithm assumes that the motor torque and reaction torque are regarded as constant, whereas they fluctuate in practice.

This paper removes the assumption and proposes a modified algorithm that estimates the reaction torque when wire-actuated robotic forceps grasp an object and compensates for the wire elongation of the forceps without any force or torque sensor. In the new algorithm, the conventional Reaction Force Observer (RFOB) [11] is used to estimate an external torque that includes not only the reaction torque but also the acceleration of the wire elongation. The wire elongation is then directly obtained from the differential equation of wire elongation.

In order to obtain the reaction torque, the acceleration of wire elongation is estimated by solving an optimization problem for a numerical solution of the differential equation of wire elongation using a gradient descent method. Thus, the reaction torque is derived by subtracting the acceleration of wire elongation from the external torque estimated by the RFOB.

The algorithm was integrated with a simulator and validated in simulation. By compensating for the estimated wire elongation, the tracking accuracy of grasping jaws of the forceps was improved, and the reaction torque was more accurately estimated than by our previous algorithm. Hence, the effectiveness of the modified algorithm was verified.

\section{MODEL OF ROBOTIC FORCEPS}

A simplified model of the wire-actuated forceps is shown in Fig. 1, where $I_{m}$ is a moment of inertia of the motor pulley, $\theta_{m}$ is a rotation angle of the motor pulley, $r_{m}$ is a radius of the motor pulley, $f$ is a tension of the wire, $k$ is a coefficient of elasticity of the wire, $x$ is an amount of elongation of the wire, $I_{j}$ is a moment of inertia of the jaw, $\theta_{j}$ is a rotation angle of the jaw, $r_{j}$ is a radius of the jaw, $\tau_{m}$ is a motor input torque, $\tau_{w}$ is a reaction torque, $\tau_{F_{j}}$ is a torque caused by coulomb friction of the jaw and $\tau_{F_{m}}$ is a torque caused by 
coulomb friction of the motor pulley. It should be noted that $\theta_{j}$ is not measurable in the actual forceps, although $\theta_{m}$ is measurable.

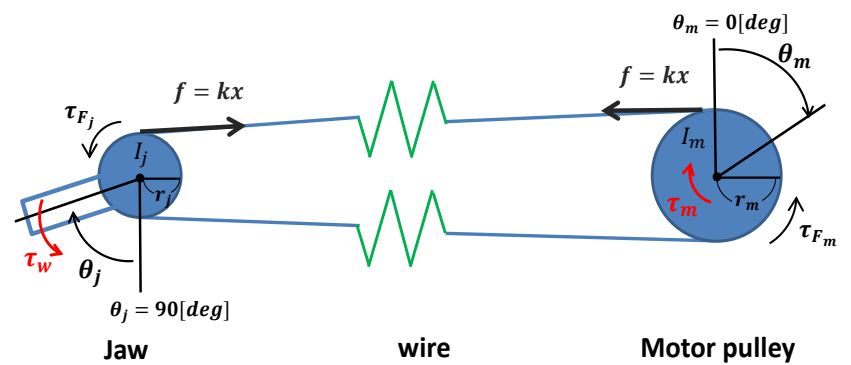

Fig. 1. Simplified model of the wire-actuated forceps.

Equations of motion of the motor pulley and jaw model are described as follows with subscripts $m$ and $j$ referring to the motor pulley and jaw respectively.

$$
\begin{gathered}
I_{m} \ddot{\theta}_{m}=\tau_{m}-D_{m} \dot{\theta}_{m}-\tau_{F_{m}}-\tau_{i n t}-r_{m} k x \\
I_{j} \ddot{\theta}_{j}=r_{j} k x-\left(\tau_{F_{j}}+\tau_{w}\right)
\end{gathered}
$$

where $D_{m}$ is a viscosity coefficient and $\tau_{\text {int }}$ is a torque caused by internal interference force such as centrifugal force.

Since the elasticity of the wire is taken into account, the amount of wire elongation is as follows.

$$
x=r_{m} \theta_{\mathrm{m}}-r_{j} \theta_{j}
$$

Thus, the following equation holds.

$$
\ddot{x}=r_{m} \ddot{\theta}_{m}-r_{j} \ddot{\theta}_{j}
$$

The following equation, obtained from (1), (2), and (4), represents the dynamics of the motor pulley, where the behaviors of the jaw and wire elongation are both taken into account.

$$
\begin{aligned}
\left(I_{m}+\frac{r_{m}^{2}}{r_{j}^{2}} I_{j}\right) \ddot{\theta}_{m}= & \tau_{m}-\left\{\frac{r_{m}}{r_{j}} \tau_{F_{j}}+D_{m} \dot{\theta}_{m}+\tau_{F_{m}}+\tau_{\text {int }}\right\}- \\
& \frac{r_{m}}{r_{j}}\left(\tau_{w}-\frac{I_{j}}{r_{j}} \ddot{x}\right)
\end{aligned}
$$

From (1), (2) and (4), the following differential equation for wire elongation is derived.

$$
\begin{gathered}
\ddot{x}+k\left(\frac{r_{m}^{2}}{I_{m}}+\frac{r_{j}^{2}}{I_{j}}\right) x=\frac{r_{m}}{I_{m}}\left\{\tau_{m}-D_{m} \dot{\theta}_{m}-\tau_{F_{m}}-\tau_{\text {int }}\right\}+ \\
\frac{r_{j}}{I_{j}}\left\{\tau_{F_{j}}+\tau_{w}\right\}
\end{gathered}
$$

\section{Previous Algorithm}

\section{A. Problem of the Conventional RFOB}

In order to estimate a reaction torque, as shown in Fig. 2, consider to apply the conventional reaction force observer (RFOB) [11] to (5), where $I=I_{m}+\frac{r_{m}^{2}}{r_{j}^{2}} I_{j}, \tau_{l}^{\prime}=\frac{r_{m}}{r_{j}} \tau_{F_{j}}+$ $D_{m} \dot{\theta}_{m}+\tau_{F_{m}}+\tau_{\text {int }}+\frac{r_{m}}{r_{j}}\left(\tau_{w}-\frac{I_{j}}{r_{j}} \ddot{x}\right), I_{a}^{r e f}$ is a current level reference, $K_{t}$ is a real value of torque coefficient, $K_{t n}$ is a nominal value of torque coefficient, $I_{n}$ is a nominal value of moment of inertia $I$ and $g_{r}$ is a value of the cutoff frequency of lowpass filter. Basic idea of the RFOB is to estimate an external torque from the difference between the input torque to the motor and the output torque which is obtained from the real machine.

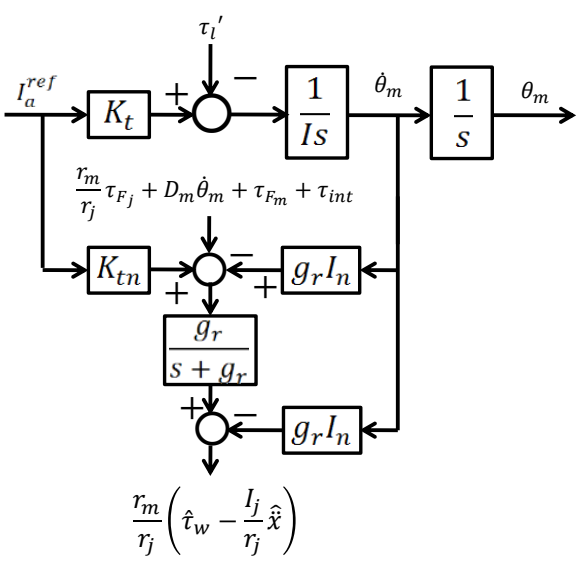

Fig. 2. Architecture of conventional RFOB.

As seen in Fig. 2, in this case, the RFOB estimates the external torque that includes both the reaction torque $\tau_{w}$ and the torque generated by the acceleration of wire elongation $\ddot{x}$. Since the acceleration of wire elongation is unknown, the reaction torque $\tau_{w}$ cannot be obtained using the conventional RFOB. Therefore, instead of estimating a reaction torque directly, consider to estimate a tension of the wire using the RFOB. Our previous algorithm [10] is described as follows.

\section{B. Estimation of the Wire Tension}

Instead of estimating a reaction torque directly, as shown in Fig. 3, consider to estimate a tension of the wire $f$, namely $k x$, using the RFOB applied to the system represented in (1), where $\tau_{l}=D_{m} \dot{\theta}_{m}+\tau_{F_{m}}+\tau_{\text {int }}+r_{m} k x$.

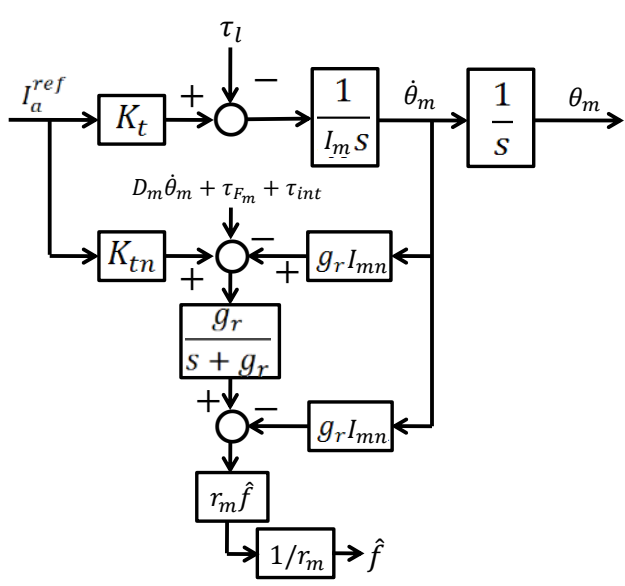

Fig. 3. Estimation of tension of wire.

\section{Analytical Solution of the Wire Elongation $x_{s}$}

Analytical solution of the nonhomogenous equation (6) is hard to obtain. However, under the assumption that the fluctuations of the motor torque $\tau_{m}$ and the reaction torque $\tau_{w}$ are negligible (i.e., $\tau_{m}$ and $\tau_{w}$ are regarded as constant), the analytical solution $x_{s}$ of (6) in the stationary state is given as follows:

$$
x_{s}=\frac{\frac{r_{m}}{I_{m}}\left(\tau_{m}-D_{m} \dot{\theta}_{m}-\tau_{F m}-\tau_{i n t}\right)+\frac{r_{j}}{I_{j}}\left(\tau_{F_{j}}+\tau_{w}\right)}{k\left(\frac{r_{m}^{2}}{I_{m}}+\frac{r_{j}^{2}}{I_{j}}\right)}
$$

\section{Estimation of the Reaction Torque $\hat{\tau}_{w}$}

To obtain an estimated reaction torque $\hat{\tau}_{w}$, substitute the 
estimated tension of the wire $\hat{f}$ into (8), which is reformulated from (7) by considering $\hat{f}=k x_{s}$.

$$
\begin{gathered}
\hat{\tau}_{w}=\hat{f}\left(\frac{r_{m}^{2}}{r_{j}} \frac{I_{j}}{I_{m}}+r_{j}\right)-\tau_{F_{j}}-\frac{r_{m}}{r_{j}} \frac{I_{j}}{I_{m}}\left(\tau_{m}-D \dot{\theta}_{m}-\right. \\
\left.\tau_{F_{m}}-\tau_{\text {int }}\right)
\end{gathered}
$$

\section{E. Estimation of the Wire Elongation $\hat{x}$}

The estimated wire elongation $\hat{x}$ is obtained by substituting the estimated reaction torque $\hat{\tau}_{w}$ into (7) as follows.

$$
\hat{x}=\frac{\frac{r_{m}}{I_{m}}\left(\tau_{m}-D_{m} \dot{\theta}_{m}-\tau_{F_{m}}-\tau_{i n t}\right)+\frac{r_{j}}{I_{j}}\left(\tau_{F_{j}}+\hat{\tau}_{w}\right)}{k\left(\frac{r_{m}^{2}}{I_{m}}+\frac{r_{j}^{2}}{I_{j}}\right)}
$$

\section{F. Compensation for the Wire Elongation}

Substitute the estimated amount of wire elongation $\hat{x}$ of (9) into equation (10), which is reformulated from (3). The rotation angle $\theta_{i}(i=j, m)$ with the corresponding subscripts is replaced with the target angle $\theta_{i}^{\text {ref }}(i=j, m)$.

$$
\theta_{m}^{r e f}=\frac{r_{j} \theta_{j}^{r e f}+\hat{x}}{r_{m}}
$$

The estimated wire elongation $\hat{x}$ to be compensated is included in the target angle $\theta_{m}^{r e f}$. Hence, the tracking ability of the grasping motion is improved.

\section{G. Problem with the Previous Algorithm}

As mentioned in Section III-C, the previous algorithm assumed that the fluctuations of the motor torque $\tau_{m}$ and reaction torque $\tau_{w}$ are negligible. However, this assumption is seldom valid in practice since surgery continuously requires grasping motions.

\section{IMPROVEMENT OF THE ALGORITHM}

To solve the problem with the previous algorithm, a new algorithm is proposed here without making any assumptions.

\section{A. Estimation of the External Torque $\hat{\tau}_{e x}$}

In the new algorithm, we apply the RFOB to the system expressed in (5) instead of solely to the system introduced in (1). Namely, the block diagram shown in Fig. 2 is adopted daringly. The estimated external torque is denoted $\hat{\tau}_{\text {ex }}$ and expressed as follows.

$$
\hat{\tau}_{e x}=\frac{r_{m}}{r_{j}}\left(\tau_{w}-\frac{I_{j}}{r_{j}} \ddot{x}\right)
$$

As seen in (11), the estimated external torque $\hat{\tau}_{\text {ex }}$ includes both the reaction torque $\tau_{w}$ and the torque generated by the acceleration of wire elongation $\ddot{x}$.

\section{B. Estimated Wire Elongation $\hat{x}$}

Equation (6) is reformulated to describe the wire elongation.

$$
x=\frac{\frac{r_{m}}{I_{m}}\left(\tau_{m}-D_{m} \dot{\theta}_{m}-\tau_{F m}-\tau_{\text {int }}\right)+\frac{r_{j}}{I_{j}} \tau_{F_{j}}+\frac{r_{j}}{I_{j}}\left(\tau_{w}-\frac{I_{j}}{r_{j}} \ddot{x}\right)}{k\left(\frac{r_{m}^{2}}{I_{m}}+\frac{r_{j}^{2}}{I_{j}}\right)}
$$

The estimated wire elongation is denoted as follows by substituting the estimated torque (11) multiplied by $r_{j}^{2} / r_{m} I_{j}$ into (12).

$$
\hat{x}=\frac{\frac{r_{m}}{I_{m}}\left(\tau_{m}-D_{m} \dot{\theta}_{m}-\tau_{F m}-\tau_{i n t}\right)+\frac{r_{j}}{I_{j}} \tau_{F_{j}}+\frac{r_{j}^{2}}{r_{m} I_{j}} \hat{\tau}_{e x}}{k\left(\frac{r_{m}^{2}}{I_{m}}+\frac{r_{j}^{2}}{I_{j}}\right)}
$$

In this way, the wire elongation $\hat{x}$ is estimated, although the reaction torque $\tau_{w}$ and the acceleration of the wire elongation $\ddot{x}$ themselves are unknown.

\section{Estimation of the Acceleration of the Wire Elongation $\ddot{x}$}

The other way to determine the wire elongation is through numerical integration. Equation (6) is reformulated as follows, introducing the subscript $n$ to indicate the numerical method.

$$
\begin{aligned}
\ddot{x}= & -k\left(\frac{r_{m}^{2}}{I_{m}}+\frac{r_{j}^{2}}{I_{j}}\right) x+\left\{\frac{r_{m}}{I_{m}}\left(\tau_{m}-D_{m} \dot{\theta}_{m}-\tau_{F_{m}}-\tau_{\text {int }}\right)+\right. \\
& \left.\frac{r_{j}}{I_{j}} \tau_{F_{j}}\right\}+\left(\frac{r_{j}}{I_{j}} \tau_{w}-\ddot{x}\right)+\ddot{x}
\end{aligned}
$$

Note that the acceleration of the wire elongation $\ddot{x}$ was subtracted from and added to the right side of (14) in order to use the external torque estimated by the RFOB. However, $\ddot{x}$ is an unknown variable. Therefore, we consider estimating $\ddot{x}$ by comparing the wire elongation $\hat{x}$ given in (13) and the numerical solution of (14). To this end, $\ddot{x}$ is replaced by a parameter $\alpha$ and (14) is rewritten as follows:

$$
\ddot{x}=-A x+B+C \hat{\tau}_{e x}+\alpha
$$

where $\quad A=k\left(\frac{r_{m}^{2}}{I_{m}}+\frac{r_{j}^{2}}{I_{j}}\right) \quad, \quad B=\left\{\frac{r_{m}}{I_{m}}\left(\tau_{m}-D_{m} \dot{\theta}_{m}-\tau_{F_{m}}-\right.\right.$ $\left.\left.\tau_{\text {int }}\right)+\frac{r_{j}}{I_{j}} \tau_{F_{j}}\right\}$ and $C=\frac{r_{j}^{2}}{r_{m} I_{j}}$.

Since $\alpha$ is the only unknown variable, the numerical solution $x_{n}$ of (15) is a function of $\alpha$. If $\alpha$ is an equivalent value of $\ddot{x}$, the numerical solution $x_{n}$ is equivalent to $\hat{x}$ given in (13). In other words, if $x_{n}$ is identical to $\hat{x}$ given in (13), then $\alpha$ is also identical to the correct value of the acceleration of wire elongation $\ddot{x}$.

In order to evaluate an error between $\hat{x}$ and $x_{n}$, the cost function $E$ is defined as follows.

$$
E=\frac{1}{2}\left(\hat{x}-x_{n}(\alpha)\right)^{2}
$$

Then, the problem to estimate the acceleration of wire elongation $\ddot{x}$ reduces to an optimization problem to find $\alpha$ which minimizes $E$.

To numerically integrate (15), the simple Euler method is employed with the intension to reduce the computation time as much as possible. The numerical integration procedure employing the simple Euler method is formulated as follows, where subscript $h$ and $k$ are the step size and the index number of the repetition of the update, respectively.

$$
\begin{aligned}
& \dot{x}_{n_{k+1}}=\dot{x}_{n_{k}}+h\left(-A x_{n_{k}}+B+C \hat{\tau}_{e x}+\alpha_{k}\right) \\
& x_{n_{k+1}}=x_{n_{k}}+h \dot{x}_{n_{k}}
\end{aligned}
$$

Considering the recurrence relation, (17) is reformulated as follows:

$$
\begin{aligned}
& x_{n_{k+1}}=x_{k}+h \dot{x}_{n_{k-1}}+h^{2}\left(-A x_{k-1}+B+C \hat{\tau}_{e x}+\right. \\
& \left.\quad \alpha_{k-1}\right)
\end{aligned}
$$


Concerning the optimization problem introduced in (16), the gradient descent method is used to find an optimal parameter $\alpha_{\text {opt }}$ also with the intention to reduce the computation time. The parameter update law of the gradient descent method is given as follows:

$$
\begin{gathered}
\alpha_{k+1}=\alpha_{k}-\eta \frac{\partial E}{\partial \alpha}=\alpha_{k}-\eta \frac{\partial E}{\partial x_{n}} \frac{\partial x_{n}}{\partial \alpha} \\
=\alpha_{k}+\eta h^{2}\left(\hat{x}-x_{n}\right)
\end{gathered}
$$

where $\eta$ is the step size used to update $\alpha$ until $\alpha_{o p t}$ is found and is usually chosen in the range $0<\eta \leq 1$.

The correct value of the acceleration of the wire elongation $\ddot{x}$ is available when the optimal parameter $\alpha_{\text {opt }}$ is found. Let $T$ denote the sampling time of RFOB, and let $h$ satisfy $h \ll T$. Then, by solving the optimization problem for each sampling time, $\alpha_{\text {opt }}$ for each sampling time can be obtained.

\section{Estimation of the Reaction Torque $\hat{\tau}_{w}$}

By substituting the identified $\hat{\ddot{x}}$ into equation (20), reformulated from (11), the reaction torque is finally estimated.

$$
\hat{\tau}_{w}=\frac{r_{j}}{r_{m}} \hat{\tau}_{e x}+\frac{I_{j}}{r_{j}} \hat{\ddot{x}}=\frac{r_{j}}{r_{m}} \hat{\tau}_{e x}+\frac{I_{j}}{r_{j}} \alpha_{o p t}
$$

\section{E. Compensation for the Wire Elongation}

Apart from the estimation of the reaction torque, the compensation for wire elongation is implemented by pulling the amount of wire elongation $\hat{x}$ given in (13) excessively with the motor pulley. The target rotation angle of the motor pulley $\theta_{m}^{r e f}$ is given by (10).

\section{SIMULATION}

To validate the improved algorithm, we simulated the behavior of a simplified model of the wire-actuated robotic forceps illustrated in Fig. 1. The simulation was carried out in MATLAB/Simulink.

\section{A. System Architecture}

The simplified model has an input voltage $V$ and an output rotation angle of the motor pulley $\theta_{m}$. The target rotation angle $\theta_{m}^{r e f}$ is calculated in the procedure of the proposed algorithm, and the voltage is controlled by a PID controller. To implement the proposed algorithm, the reference current $I_{\alpha}^{r e f}$ in the RFOB is calculated from the input voltage $V$ divided by the armature resistance of the motor $\Omega_{m}$.

\section{B. Parameter Setup}

TABLE I: PHYSICAL PARAMETERS
\begin{tabular}{|c|c|}
\hline Parameter & Value \\
\hline$r_{m}, r_{j}$ & $2.00 \times 10^{-3}, 1.4 \times 10^{-3} \mathrm{~m}$ \\
\hline$I_{m}, I_{m n}$ & $3.18 \times 10^{-8} \mathrm{kgm}^{2}$ \\
\hline$I_{j}$ & $6.85 \times 10^{-8} \mathrm{kgm}^{2}$ \\
\hline$K_{t}, K_{t n}$ & $1.35 \times 10^{-2} \mathrm{Nm} / \mathrm{A}$ \\
\hline$g_{r}$ & $70 \mathrm{~Hz}$ \\
\hline$k$ & $2.60 \times 10^{4} \mathrm{~N} / \mathrm{m}$ \\
\hline$\tau_{F_{m}}, \tau_{F_{j}}$ & $4.62 \times 10^{-7}, 4.62 \times 10^{-9} \mathrm{Nm}$ \\
\hline$D$ & $1.16 \times 10^{-4} \mathrm{kgm}^{2} / \mathrm{s}$ \\
\hline
\end{tabular}

The physical parameters of the simulation are given in Table I. Note that the error between the real value and the nominal value is disregarded in order to run the simulation under ideal conditions.

\section{Simulation Setup}

The reaction torque was added in two ways in the simulations, using the previous algorithm and the new algorithm, for comparison. In the first of two simulations, the reaction torque in the steady state was set as constant to satisfy the assumption made by the previous algorithm. In the second simulation, the reaction torque in the steady state was given as a pulse wave, and the assumption made by the previous algorithm was not satisfied. The relationship between the sampling time $T$ and the step size $h$ was set as $h=T / 2$ so that $\alpha$ is updated twice for every sampling time.

\section{Simulation 1: Without Rapid-Transient Reaction Torque}

1) The initial angle of the jaw is set as $\theta_{j}=90 \mathrm{deg}$, and the target rotation angle of the jaw is set as $\theta_{j}^{\text {ref }}=10 \mathrm{deg}$.

2) From 0 to $5 \mathrm{~s}$ : From the initial angle of the jaw of $90 \mathrm{deg}$, the jaw is controlled to reach to the target rotation angle of $10 \mathrm{deg}$ following a ramp function by winding the wire with a motor pulley.

3) From 5 to $10 \mathrm{~s}$ : The rotation angle of the jaw $\theta_{j}$ is controlled to keep the target rotation angle $\theta_{j}^{\text {ref }}$ throughout the simulation.

4) From 10 to $15 \mathrm{~s}$ : The load on the joint bearing as the reaction torque $\tau_{w}$ is added gradually from 0 to 0.002 $\mathrm{Nm}$ following a ramp function.

5) From 15 to $20 \mathrm{~s}$ : Constant torque $\tau_{w}=0.002 \mathrm{Nm}$ is added until the end of the simulation.

The results of Simulation 1 are shown in Fig. 4 and Fig. 5.

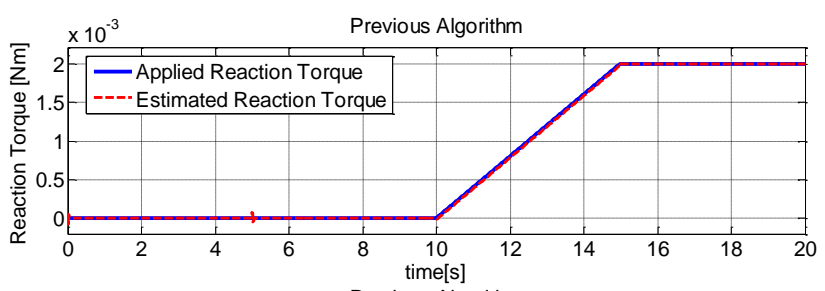

Previous Algorithm

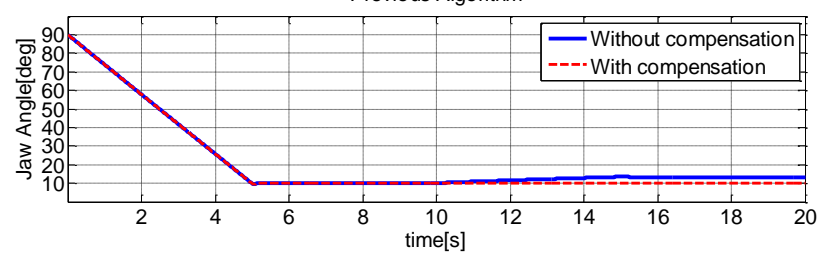

Fig. 4. Results obtained with the previous algorithm in simulation 1.
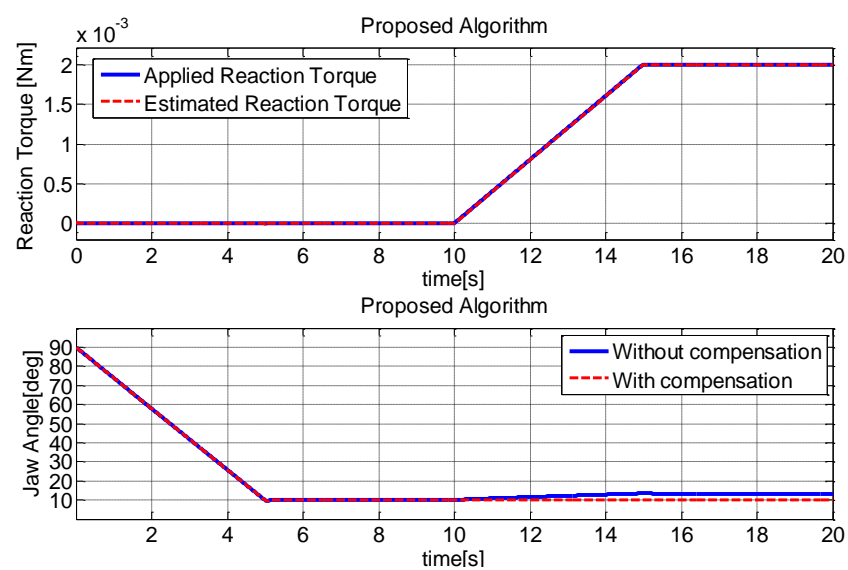

Fig. 5. Results obtained with the improved algorithm in simulation 1. 
The results show that there is no notable difference between the results obtained with the two algorithms. Both algorithms ideally estimated $\tau_{w}$ and compensated for the wire elongation $x$ so that the jaw angle achieved the target rotation angle $\theta_{j}^{r e f}=10 \mathrm{deg}$, even after the reaction torque was added.

\section{E. Simulation 2: With Rapidly Transient Reaction Torque}

1) The initial angle of the jaw is set as $\theta_{j}=90 \mathrm{deg}$, and the target rotation angle of the jaw is set as $\theta_{j}^{\text {ref }}=10 \mathrm{deg}$.

2) and 3) From 0 to $10 \mathrm{~s}$ : The operations are the same as in simulation 1.

4) From 10 to $20 \mathrm{~s}$ : A pulse-shaped reaction torque with a cycle of $5 \mathrm{~s}$ and magnitude of $0.002 \mathrm{Nm}$ is added.

The results of Simulation 2 are shown in Fig. 6 and Fig. 7.
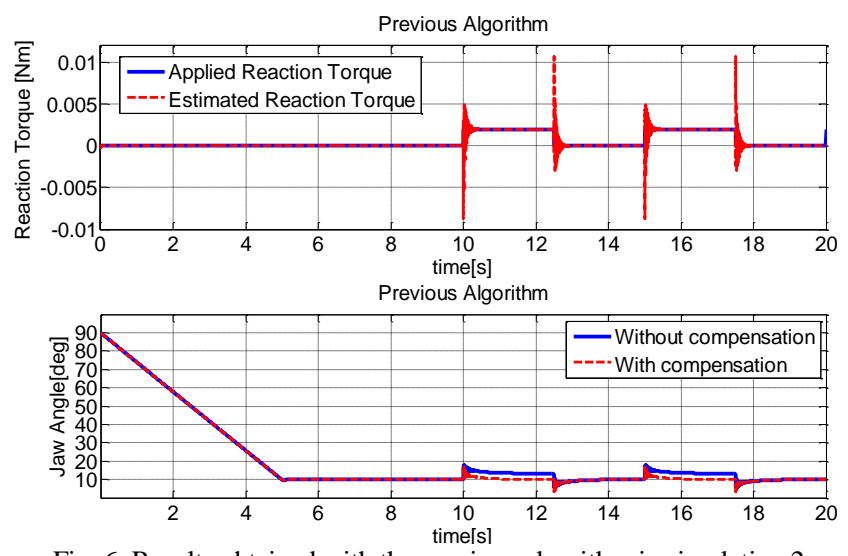

Fig. 6. Results obtained with the previous algorithm in simulation 2.
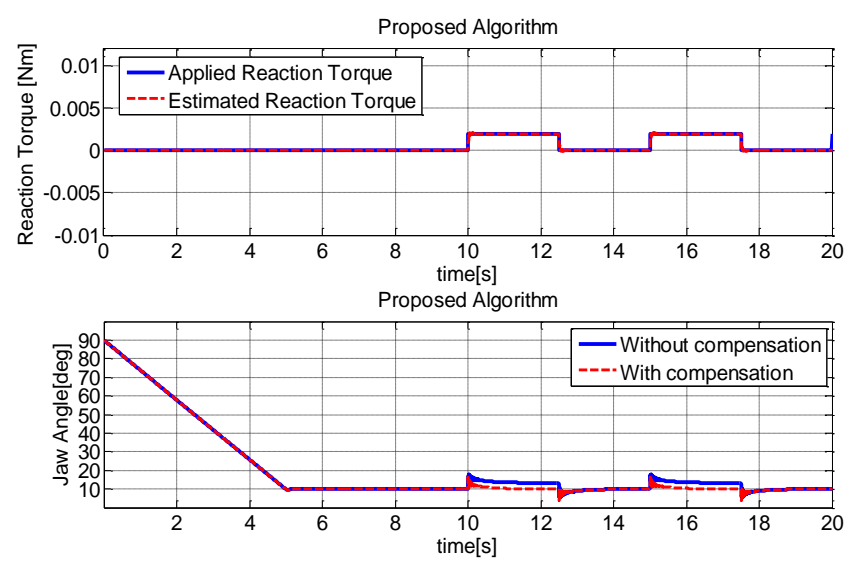

Fig. 5. Results obtained with the improved algorithm in simulation 2.

The compensation for the wire elongation appeared similar in the two results. However, large estimation error is seen in the reaction torque estimated by the previous algorithm when the reaction torque transiently changed (at 10, 12.5, 15 and $17.5 \mathrm{~s}$ ), whereas such estimation error is not seen in the reaction torque estimated by the improved algorithm. The reaction torque estimated by the improved algorithm coincides well with the added reaction torque. Hence, we conclude the effectiveness of the improved algorithm.

\section{CONCLUSIONS}

A new algorithm that estimates the reaction torque and compensates for wire elongation of wire-actuated robotic forceps was proposed. The new algorithm did not require the assumption made by the previous algorithm, and improved the accuracy of the estimated reaction torque. As the next step, to confirm the effectiveness of the new algorithm, experimental works using the two algorithms are needed for comparison.

\section{ACKNOWLEDGMENT}

The authors would like to thank for Grants-In-Aid for Scientific Research and Shinnosuke Shibata for his assistance.

\section{REFERENCES}

[1] Intuitive Surgical. [Online]. Available: http://www.intuitivesurgical.com/jp/

[2] T. Lens and O. von Stryk, "Design and dynamics model of a lightweight series elastic tendon-driven robot arm," in Proc. the 2013 IEEE Int. Conf. on Robotics and Automation, 2013, pp. 4512-4518.

[3] S. Can, B. Jensen, E. Dean-Leon, C. Staub, A. Knoll, A. Fiolka, A. Schneider, A. Meining, and H. Feussner, "Kinematics, control and workspace analysis of a Bowden wire actuated manipulator for minimally invasive single-port surgery," in Proc. the 2012 IEEE Int. Conf. on Robotics and Biomimetics, 2012, pp. 848-853.

[4] K. Ikuta, T. Hasegawa, and S. Daifu, "Hyper redundant miniature manipulator "'Hyper Finger' for remote minimally invasive surgery in deep area," in Proc. the 2003 IEEE Int. Conf. on Robotics \& Automation, 2003, pp. 1098-1102.

[5] K. Ikuta, M. Hasegawa, and H. Goto, "Total system of hyper finger for remote minimally invasive surgery (The 9th report) proposal and experimental verification of safety operation strategies," Journal of the Japan Society of Computer Aided Surgery, vol. 9, no. 3, pp. 210-211, 2007.

[6] Y. Tsukahara, Y. Yoshimura, E. Kobayashi, and I. Sakuma, "Force sensing of two degrees-of-freedom bending forceps," in Proc. Welfare, Wellbeing, Life Support 2010, 2010, p. 454.

[7] U. Seibold, B. Kubler, and G. Hirzinger, "Prototype of instrument for minimally invasive surgery with 6-axis force sensing capability," in Proc. 2005 IEEE Int. Conf. on Robotics and Automation, pp. 496-501, 2005.

[8] N. Zemiti, G. Morel, T. Ortmaier, and N. Bonnet, "Mechatronic Design of a new robot for force control in minimally invasive surgery," IEEE/ASME Trans. on Mechatronics, vol.12, no. 2, pp.143-153, 2007.

[9] C. Ishii, H. Mikami, T. Nakakuki, and H. Hashimoto, "Bilateral control for remote controlled robotic forceps system with time varying delay," in Proc. the 4th International Conference on Human System Interactions, pp. 330-335, 2011.

[10] C. Ishii and G. Komada, "Estimation of reaction torque and compensation for elongation of wire for wire actuated robotic forceps," in Proc. the 19th World Congress of the International Federation of Automatic Control 2014, August 2014, pp. 7233-7238.

[11] K. Ohnishi, M. Shibata, and T. Murakami, "Motion control for advanced mechatronics," IEEE/ASME Transactions on Mechatronics, vol. 1, no. 1, pp. 56-67, 1996.

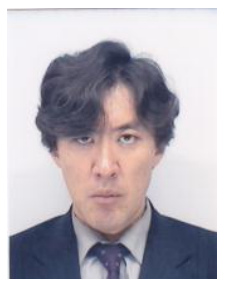

Chiharu Ishii received his $\mathrm{PhD}$ degree in mechanical engineering from Sophia University, Japan in 1997. From 2002 to 2009, he was an assistant professor with Kogakuin University. Currently, he is a professor a the Department of Mechanical Engineering, Hosei University, Japan. His research interests are in medical robotics, assistive technology and robust control. He is a member of JSME, SICE, RSJ, IEEJ and IEEE.

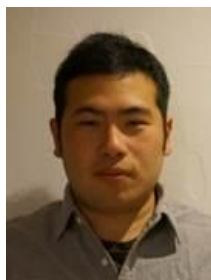

Gakuto Komada received his BE degree in mechanical engineering from Hosei University, Japan in 2011. Currently, he is a master course student at Graduate School of Engineering, Hosei University, Japan. His research interest is in medical robotics. 